André LIEBICH \& Svetlana YAKIMOVICH (dir.), From Communism to AntiCommunism: Photographs from the Boris Souvarine Collection at the Graduate Institute, Geneva, Genève : Graduate Institute Publications, Coll. eLivres de 1'Institut, 2016, n ${ }^{\circ} 6,160$ p. <http://books.openedition.org/iheid/6440>

\title{
SOPHIE COEURÉ
}

\section{Université de Paris, ICT}

Boris Souvarine (1895-1984) reste sans doute, avec Victor Serge (1890-1947), la figure la plus connue de la première génération de militants européens ralliés avec enthousiasme à l'Octobre bolchevique et à l'organisation d'une révolution mondiale par l'Internationale communiste, passés dans l'opposition dans les années 1920, et dès lors exclus et bannis d'Union soviétique. L'itinéraire politique et personnel de l'auteur de Staline. Aperçu historique du bolchevisme paru en 1935, est bien connu, notamment par la biographie que lui a consacrée Jean-Louis Panné en 1993. André Liebich, professeur au Graduate Institute of International Studies de Genève, évoque en ouverture de cet ouvrage l'engagement d'un homme passionné, au fort tempérament («bitter and acidic »). Il rappelle sa fréquentation des bords du lac Léman, notamment lors d'un colloque préparant le cinquantième anniversaire de la révolution d'Octobre auquel participait également « Big Boris », Boris Nikolaevsky (1887-1966), figure de l'émigration menchevique à Berlin, puis à Paris et aux États-Unis. Comme Souvarine, il avait été le correspondant à l'étranger de l'Institut Marx-Engels, qui rassemblait à Moscou la documentation fondatrice du mouvement socialiste mondial, avant de devenir historien et acteur clé de la collecte archivistique de l'exil antistalinien.

Svetlana Yakimovich, responsable du fonds Souvarine au Graduate Institute of International Studies de Genève, retrace le processus d'arrivée du fonds dans les Archives de l'institution. Après la première collecte d'archives (dont une partie de celles de Trotski) et d'ouvrages pour l'Institut d'histoire sociale d'Amsterdam et sa branche de Paris dans les années 1930, Nikolaevsky et Souvarine avaient dû faire face au traumatisme du pillage nazi (rapidement évoqué, et qui toucha aussi les collections personnelles de Souvarine), à la guerre froide et à la menace d'une invasion soviétique redoutée de la France, ainsi qu'aux difficultés financières personnelles d'une vie en émigration. Nikolaevsky devint le curateur des archives et des livres qu'il avait vendus à la Hoover Institution at Stanford University. Par l'intermédiaire de Jacques Freymond, qui développait des études sur les Internationales et le communisme européen, Souvarine envoya progressivement vers Genève des milliers d'ouvrages, des brochures et de la documentation personnelle, mais aussi des archives qui avaient été confiées à ses soins par d'autres émigrés. Dans le même temps, il refondait à Paris l'Institut d'histoire sociale et accumulait une nouvelle et riche documentation sur l'URSS. Après le décès de Souvarine en 1985, son épouse a versé un dernier lot d'archives.

Cet ouvrage n'a toutefois pas pour objectif de documenter l'histoire passionnante et peu connue de cette « contre-archive » de la révolution russe et du socialisme antistalinien, mais de proposer au lecteur, sur papier glacé et en ligne, un choix de 
fleurons iconographiques de la collection Souvarine, commentés par les meilleurs historiens. La section la plus fournie illustre les manifestations et commémorations révolutionnaires de 1917 à 1920 et les débuts de la III Internationale, avec un rare ensemble de photographies du second congrès du Komintern tenu à l'été 1920. La deuxième partie propose une plongée dans la vie quotidienne des Soviétiques des années 1930-1931 : vie rurale, éducation du peuple, pionniers en Asie centrale, etc. La troisième partie est une sélection du dossier de la presse internationale rassemblé par Souvarine après la mort de Staline en mars 1953. Enfin, la dernière section permet d'évoquer l'anticommunisme de guerre froide à travers quelques affiches, tracts et caricatures.

L'édition est peu soigneuse en ce qui concerne les documents eux-mêmes : on ne trouvera ni cotes relatives au fonds genevois, ni explications sur l'origine supposée des photographies (la publication de certaines annotations au dos permet de comprendre qu'elles viennent parfois des agences de presses soviétiques, ou encore de la propagande du Komintern vers l'Allemagne par exemple), ni commentaires sur leur caractère rare ou iconique. On ne trouvera pas de précisions sur l'origine avérée des extraits de presse (les tirages par exemple), ou sur la conception et la diffusion des affiches, tracts et caricatures de guerre froide, produites pour l'essentiel par le mouvement Paix et Liberté, dans le contexte de la diplomatie culturelle des années 1950.

Il reste un bel ouvrage, une plongée visuelle éclectique et intéressante dans l'histoire de la révolution russe et de l'Union soviétique, par un choix pertinent d'images bien situées et commentées par les notices accompagnées d'une orientation bibliographique. 